\title{
CALIDAD POSCOSECHA DE CULTIVARES DE MANGO DE MADURACIÓN TEMPRANA, INTERMEDIA Y TARDÍA
}

\section{POSTHARVEST QUALITY OF MANGO CULTIVARS OF EARLY, MIDDLE AND LATE SEASONS}

\author{
Jorge Siller-Cepeda $^{1 *}$, Dolores Muy-Rangel ${ }^{1}$, Manuel Báez-Sañudo ${ }^{1}$, Evelia Araiza-Lizarde ${ }^{1}$ y \\ Adolfo Ireta-Ojeda ${ }^{2}$
}

\begin{abstract}
${ }^{1}$ Centro de Investigación en Alimentación y Desarrollo A. C.-Unidad Culiacán. Carretera a Eldorado Km 5.5, Apdo. Postal 32-A. 80129, Culiacán, Sinaloa, México. ${ }^{2}$ Campo Experimental Valle de Culiacán, Centro de Investigación Regional del Noroeste, Instituto Nacional de Investigaciones Forestales, Agrícolas y Pecuarias.

*Autor para correspondencia (jsiller@ciad.edu.mx)
\end{abstract}

\section{RESUMEN}

Se evaluó el comportamiento poscosecha de 12 cultivares de mango (Mangifera indica L.) cinco de maduración temprana ('Edward', 'Diplomático', 'Ah-Ping', 'Van Dyke', 'Haden'), tres de intermedia ('Manila Rosa', 'Tommy Atkins', 'Kent') y cuatro tardías ('Osteen', 'Palmer', 'Fabián', 'Keitt'); los mangos 'Haden', 'Tommy Atkins', 'Kent' 'Keitt' se consideraron testigos comerciales. Los frutos se almacenaron bajo simulación de mercadeo a $20^{\circ} \mathrm{C}$ y $87 \% \mathrm{HR}$ por $12 \mathrm{~d}$, y durante este periodo se hicieron evaluaciones físicas, químicas y fisiológicas. El tamaño y peso de los frutos varió desde 583 g ('Osteen') hasta poco menos de 250 g ('Diplomático', 'Van Dyke' y 'Manila Rosa'). Los mangos de maduración tardía presentaron la menor pérdida de peso $(<4 \%)$, con excepción de 'Kent'. Al final del estudio, la mayor firmeza del fruto la mantuvieron 'AhPing' y 'Kent' con 2.3 y 2.6 kgf, respectivamente. 'Ah-Ping', 'Kent' y 'Fabián' desarrollaron un color de pulpa atractivo $\left({ }^{\circ} \mathrm{Hue}=78\right)$ y un contenido de sólidos solubles superior a $12.5{ }^{\circ}$ Brix. 'Edward', 'Ah-Ping', 'Fabián' y 'Palmer' fueron los cultivares con calidad competitiva en relación con los mangos comerciales.

Palabras clave: Mangifera indica, calidad, poscosecha, variedades.

\section{SUMMARY}

Postharvest behavior of 12 mango (Mangifera indica L.) cultivars, five of early ('Edward', 'Diplomático', 'Ah-Ping', 'Van Dyke', 'Haden'), three of middle ('Manila Rosa', 'Tommy Atkins', 'Kent') and four of late season ('Osteen', 'Palmer', 'Fabián', 'Keitt') was evaluated. Cvs. 'Haden', 'Tommy Atkins', 'Kent' 'Keitt' were considered as commercial controls. The fruits were stored simulating marketing conditions at $20{ }^{\circ} \mathrm{C}$ and $87 \% \mathrm{RH}$ during $12 \mathrm{~d}$, and physical, chemical and physiological evaluations were conducted during this period. Fruit size and weight varied from $586 \mathrm{~g}$ ('Osteen') to almost 250 g ('Diplomático', 'Van Dyke' and 'Manila Rosa'). The lowest weight loss $(<4 \%)$ was presented by all late season cultivars except 'Kent'. At the end of the storage, fruit firmness was higher in 'Ah-Ping' and 'Kent' with 2.3 and 2.6 kgf respectively. 'Ah-Ping', 'Kent' and 'Fabián' developed an attractive flesh color $\left({ }^{\circ} \mathrm{Hue}=78\right)$ and a soluble solids content up to $12.5{ }^{\circ}$ Brix. 'Edward', 'Ah-Ping',
'Fabián' and 'Palmer' were the cultivars with competitive quality fruits compared to the controls.

Index words: Mangifera indica, quality, postharvest, varieties.

\section{INTRODUCCIÓN}

En México el cultivo del mango (Mangifera indica $\mathrm{L}$.) tiene importancia agrícola. En el 2006 se produjeron 1.7 millones de toneladas en 172 mil hectáreas, y los principales estados productores fueron Sinaloa, Guerrero, Nayarit, Oaxaca, Chiapas, Veracruz y Michoacán, que aportaron $90 \%$ de la producción nacional (SIAP, 2008). En este año, la exportación de mango mexicano generó 117 millones de dólares, que representa $14.4 \%$ del total de la producción nacional (FAO, 2004), y el resto se destinó al consumo interno aparente con un consumo per capita de $16 \mathrm{~kg}$ (SICM, 2008). Los principales cultivares de mango que se producen en el país con fines de exportación son 'Kent', 'Keitt', 'Tommy Atkins' y 'Haden', y en los últimos años también 'Ataulfo', todos ellos por sus características sobresalientes de sabor, aroma y tamaño (Báez, 1998; Osuna-García et al., 2002).

El Estado de Sinaloa se caracteriza por producir frutos con calidad de exportación, ya que las condiciones climatológicas y la calidad del suelo de cultivo favorecen su desarrollo. Con el propósito de aprovechar sus cualidades, en los últimos años se ha promovido la búsqueda de cultivares que presenten rendimiento alto, resistencia a enfermedades y larga vida de anaquel. A partir de 1984 en Sinaloa se han establecido clones y selecciones de mango de diversas regiones del mundo con el fin de formar un banco de germoplasma de este cultivo; la mayoría de los estudios se han enfocado a evaluar las 
características agronómicas y de adaptabilidad (Ireta y Guzmán, 2002). Sin embargo, la información de materiales de mango promisorios en calidad y comportamiento poscosecha es tan importante como los datos fenotípicos y de rendimiento. En este trabajo se evaluaron los cambios de calidad poscosecha de 12 cultivares de mango de maduraciones temprana, intermedia y tardía, para identificar aquellos que igualen o superen las características de calidad y vida de anaquel de los materiales comerciales cultivados.

\section{MATERIALES Y MÉTODOS}

\section{Germoplasma evaluado}

Se estudiaron 12 cultivares de mango de tres grupos de maduración: temprana ('Edward', 'Diplomático', 'AhPing', 'Van Dyke', 'Haden'; donde 'Haden' es mango comercial) que maduran de mayo a julio (en un rango térmico de $15.3-35.4{ }^{\circ} \mathrm{C}$ durante el periodo), intermedia ('Manila Rosa', 'Tommy Atkins' y 'Kent', donde 'Tommy Atkins' y 'Kent', son mangos comerciales) de julio a agosto $\left(24.5-34.7{ }^{\circ} \mathrm{C}\right)$, y tardía ('Osteen', 'Palmer', 'Fabián' y 'Keitt' donde 'Keitt' es mango comercial) de agosto a septiembre $\left(23.8-33.5^{\circ} \mathrm{C}\right)$, todos ellos ubicados en el banco de germoplasma del Instituto Nacional de Investigaciones Forestales, Agrícolas y Pecuarias (INIFAP), en Aguaruto, Sinaloa, México. De cada cultivar, se cosecharon 150 frutos en madurez fisiológica, que se redujeron a 123 frutos al seleccionarlos por peso, color, ausencia de daños y enfermedades; los frutos se lavaron con una solución acuosa de cloro $200 \mu \mathrm{L} \mathrm{L}^{-1}$, previo a su almacenamiento.

\section{Almacenamiento y variables medidas}

Los frutos se almacenaron bajo simulación de mercadeo por $12 \mathrm{~d}$ a $20{ }^{\circ} \mathrm{C}$ y $87 \%$ HR. Diariamente se determinó la pérdida de peso y respiración; cada $3 \mathrm{~d}$ se evaluó firmeza (kgf), colores interno y externo (luminosidad, cromaticidad y ángulo de matiz), $\mathrm{pH}$, acidez titulable (\% ácido cítrico), sólidos solubles totales $\left({ }^{\circ} \mathrm{Brix}\right)$ y la relación azúcar/ácido (RAA). El porcentaje de pulpa, cáscara y semilla se calculó en frutos maduros de la última fecha de evaluación.

Cuantificación de biomasa. Para cuantificar el contenido de pulpa, cáscara y semilla de los frutos se separó manualmente y se pesó cada parte en forma individual, en 10 frutos de cada cultivar, con una balanza digital Sartorius BP 4100. La proporción de cada parte se calculó con base en el peso total del fruto.
Pérdida de peso. Se registró diariamente el peso de 10 frutos desde el inicio y hasta el final de los $12 \mathrm{~d}$ de almacenamiento, para calcular el porcentaje de pérdida acumulada de peso (Muy et al., 2004).

Firmeza. Se determinó el esfuerzo necesario para penetrar la pulpa de 10 mangos sin cáscara de cada cultivar, con un puntal de $8 \mathrm{~mm}$ de diámetro en ambos lados del fruto y en tres secciones (ápice, centro y base) (Bourne, 1980). Se utilizó un penetrómetro Chatillon Modelo DFIS-50, y los resultados se expresaron en $\mathrm{kgf}(1 \mathrm{kgf}=$ $9.8 \mathrm{~N})$.

Color externo e interno. El color de la pulpa se evaluó en 20 repeticiones, en la zona más cercana a la semilla, y el color externo en la cáscara de lados opuestos del fruto y en tres secciones (ápice, centro y base) (SillerCepeda et al., 1994), con un colorímetro portátil Minolta CR-300 que registró luminosidad (L), cromaticidad (C) y ángulo de matiz ( $\left.{ }^{\circ} \mathrm{Hue}\right)$.

pH, sólidos solubles totales, acidez titulable y relación azúcar/ácido (RAA). Estas determinaciones se hicieron con las metodologías respectivas de la AOAC (1998). Para el pH se utilizó un potenciómetro digital Corning pH Meter 140. Para los sólidos solubles totales (SST) se usó un refractómetro ABBE Leica Mark II, y los resultados se expresaron en ${ }^{\circ}$ Brix. Para la acidez titulable (\% ácido cítrico) se tomaron $20 \mathrm{~mL}$ de extracto $(10 \mathrm{~g}$ de muestra en $50 \mathrm{~mL}$ de agua destilada) y se tituló con $\mathrm{NaOH} 0.1 \mathrm{~N}$ hasta pH de 8.2. La RAA se calculó al dividir los valores de los sólidos solubles totales ( $\left.{ }^{\circ} \mathrm{Brix}\right)$ entre el porcentaje de acidez titulable.

Análisis fisiológicos. La velocidad de respiración se hizo según Báez et al. (1997). Los frutos se pesaron y se colocaron en frascos de vidrio herméticos de $3.7 \mathrm{~L}$ adaptados con mangueras de entrada y salida de aire, y conectados a un tren de respiración con flujo constante de aire $\left(60 \mathrm{~mL} \mathrm{~min}^{-1}\right)$ libre de $\mathrm{CO}_{2}$. Diariamente y durante $12 \mathrm{~d}$ se tomó $1 \mathrm{~mL}$ de aire de la manguera de salida y se analizó en un cromatógrafo de gases Varian 3300 provisto con una columna de separación HayeSepQ 100/120 de $182 \mathrm{~cm}$ x $0.32 \mathrm{~cm}$ a $70{ }^{\circ} \mathrm{C}$. La identificación del $\mathrm{CO}_{2}$ se hizo con un detector de conductividad térmica operado a $170{ }^{\circ} \mathrm{C}$. Se reportaron los valores máximos y mínimos de $\mathrm{CO}_{2}$ producido del promedio de tres frutos por tratamiento en $\mathrm{mL} \mathrm{CO} \mathrm{kg}^{-1} \mathrm{~h}^{-1}$. Como estándar se usó $\mathrm{CO}_{2}$ a $0.52 \%$ preparado con nitrógeno (AGA GAS, S.A. de C.V.; Sinaloa, México).

Análisis estadístico. Para los mangos de cada época de maduración se usó un diseño completamente al azar con un factor (variedad) con 5, 3 y 4 niveles para la 
maduración temprana, intermedia y tardía, respectivamente. Las variables de respuestas de calidad fueron porcentaje de pulpa, piel, semilla, peso total y pérdida de peso; así como firmeza, $\mathrm{pH}$, sólidos solubles totales, acidez titulable y relación azúcar/ácido con 10 repeticiones; luminosidad, cromaticidad y ángulo de matiz, con 20 repeticiones, y producción de bióxido de carbono con tres repeticiones. La unidad experimental fue un fruto. Cuando los análisis de varianza detectaron diferencias significativas entre tratamientos, se aplicó la prueba de comparación de medias de Tukey $(\alpha=0.05)$, mediante el programa estadístico computacional MINITAB (2004) versión 14.0.

\section{RESULTADOS Y DISCUSIÓN}

\section{Masa de la estructura del fruto}

En la categoría de maduración temprana, los cultivares 'Edward' y 'Ah-Ping' presentaron el mayor peso y el menor porcentaje de semilla (Cuadro 1). En el grupo de madurez intermedia, 'Kent' fue el de mayor peso con $486 \mathrm{~g}$ y $75 \%$ de pulpa, mientras que 'Manila Rosa' fue el de menor peso y con mayor porcentaje de semilla y cáscara. Los materiales de maduración tardía mostraron los pesos mayores de fruto, con 'Osteen' como el más alto con 583 g, y sin diferencia significativa entre 'Fabián', 'Keitt' y 'Palmer'.

De acuerdo con la Norma Mexicana de Calidad para Mango Fresco de Exportación, 66 \% de los mangos estudiados son óptimos para comercializar en función del peso, con frutos pequeños, medianos y grandes que pueden dar calibres comerciales entre 20 (180-220 g) y ocho (450 a 550 g) (Báez, 1998). Campbell (1992) describió las características agronómicas de 80 cultivares de mango des- arrollados en Florida, EE. UU., cuyos frutos en madurez de consumo presentaron pesos y tamaños variables dentro de un mismo cultivar; por ejemplo, los mangos 'Keitt' pesaron desde 510 hasta $2000 \mathrm{~g}$, mientras que Hofman et al. (1997) reportaron entre 500 y 571 g para este mismo cultivar producido en Queensland, Auatralia, lo que indica que otros factores de producción influyen en el desarrollo del fruto.

\section{Pérdida de peso}

Los mangos evaluados se caracterizaron por mostrar una pérdida final de peso relativamente baja (entre 3 y 6 \%) que en los frutos 'Ah-Ping' y 'Manila Rosa' significó marchitamiento lo que sugiere que son de mayor sensibilidad (Muy et al., 2004; Siller-Cepeda et al., 1994); con excepción de 'Diplomático' (7.4\%), los frutos de 'Kent', 'Palmer', 'Osteen', 'Van Dyke', 'Tommy Atkins' y 'Haden' presentaron pérdidas menores a $4 \%$ (Cuadro 1).

Hidalgo et al. (1996) reportaron una pérdida de peso de $11 \%$ en frutos 'Manila' almacenados por $10 \mathrm{~d}$ a $25{ }^{\circ} \mathrm{C}$; posiblemente la temperatura de $25{ }^{\circ} \mathrm{C}$ durante el almacenamiento aceleró la transpiración del fruto y hubo así una mayor pérdida de peso, en relación con el este estudio que aquí se reporta. Lo anterior se corrobora con los datos de pérdida de peso durante el almacenamiento por $23 \mathrm{~d}$ a $12{ }^{\circ} \mathrm{C}$ reportados por los mismos autores para mangos 'Kent' (4\%) y 'Tommy Atkins' (3.2\%). Muy et al. (2004) reportaron pérdidas de peso de 4,6 y $11 \%$ en frutos de mango 'Keitt' recubiertos con películas de polietileno (Britex ${ }^{\circledR}$ ) y almacenados a $24{ }^{\circ} \mathrm{C}$ por $10 \mathrm{~d}$ a 93,64 y $52 \% \mathrm{HR}$, respectivamente, lo que indica que la humedad relativa es otra variable importante a considerar en la pérdida de peso de los frutos durante el almacenamiento.

Cuadro 1. Composición en base húmeda de pulpa, cáscara, semilla, biomasa y pérdida de peso en frutos de mango de tres grupos de maduración.

\begin{tabular}{|c|c|c|c|c|c|}
\hline Cultivar & Pulpa $(\%)^{\dagger}$ & Cáscara $(\%)^{\dagger}$ & Semilla $(\%)^{\dagger}$ & Peso total $(\mathrm{g})^{\dagger}$ & Pérdida de peso $(\%)^{\dagger \dagger}$ \\
\hline \multicolumn{6}{|c|}{ Maduración temprana } \\
\hline 'Edward' & $76 \pm 1.5 \mathrm{a}^{\dagger \dagger \dagger}$ & $17 \pm 0.8 \mathrm{~b}$ & $7 \pm 1.3 \mathrm{~b}$ & $473 \pm 27.2 \mathrm{a}$ & $6.0 \pm 0.6 \mathrm{~b}$ \\
\hline 'Ah-Ping' & $74 \pm 0.9 \mathrm{a}$ & $18 \pm 1.7 \mathrm{ab}$ & $8 \pm 1.0 \mathrm{~b}$ & $438 \pm 28.7 \mathrm{a}$ & $5.6 \pm 0.5 \mathrm{~b}$ \\
\hline 'Van Dyke' & $65 \pm 2.3 \mathrm{~b}$ & $22 \pm 1.6 \mathrm{a}$ & $13 \pm 2.2 \mathrm{a}$ & $247 \pm 30.6 \mathrm{~b}$ & $3.8 \pm 0.3 \mathrm{c}$ \\
\hline 'Haden' & $70 \pm 2.7 \mathrm{ab}$ & $21 \pm 1.8 \mathrm{a}$ & $9 \pm 1.7 \mathrm{ab}$ & $297 \pm 33.6 \mathrm{~b}$ & $4.0 \pm 0.5 \mathrm{c}$ \\
\hline \multicolumn{6}{|c|}{ Maduración intermedia } \\
\hline 'Tommy Atkins' & $76 \pm 1.5 \mathrm{a}$ & $14 \pm 0.8 \mathrm{~b}$ & $10 \pm 1.7 \mathrm{~b}$ & $375 \pm 20.3 \mathrm{~b}$ & $3.8 \pm 0.3 \mathrm{~b}$ \\
\hline 'Kent' & $75 \pm 1.4 \mathrm{a}$ & $15 \pm 1.4 \mathrm{~b}$ & $10 \pm 1.7 \mathrm{~b}$ & $486 \pm 20.5 \mathrm{a}$ & $2.5 \pm 0.2 \mathrm{c}$ \\
\hline \multicolumn{6}{|c|}{ Maduración tardía } \\
\hline 'Osteen’ & $64 \pm 2.8 \mathrm{~b}$ & $22 \pm 1.8 \mathrm{a}$ & $14 \pm 1.6 \mathrm{a}$ & $583 \pm 17.6 \mathrm{a}$ & $3.4 \pm 0.3 b$ \\
\hline 'Palmer' & $79 \pm 5.0 \mathrm{a}$ & $13 \pm 1.2 \mathrm{c}$ & $8 \pm 1.1 \mathrm{c}$ & $435 \pm 25.4 \mathrm{~b}$ & $3.5 \pm 0.3 \mathrm{~b}$ \\
\hline 'Fabián' & $70 \pm 4.3 \mathrm{ab}$ & $19 \pm 1.3 \mathrm{ab}$ & $11 \pm 1.2 \mathrm{~b}$ & $482 \pm 27.9 \mathrm{~b}$ & $4.3 \pm 0.4 \mathrm{a}$ \\
\hline
\end{tabular}




\section{Firmeza}

$\mathrm{Al}$ inicio del estudio algunos frutos presentaron valores superiores a los mínimos de madurez requeridos $\left(13.2,12.2,12.4\right.$ y $11 \mathrm{kgf} \mathrm{cm}^{-2}$, para 'Haden', 'Tommy Atkins', 'Kent' y 'Keitt', respectivamente) según la Norma Mexicana para mango (Báez, 1998). Con excepción de las variedades 'Osteen' y 'Fabián', todos los mangos presentaron estándares mínimos de firmeza para su comercialización a los 6 d de almacenamiento (Cuadro 2). Se esperaba que durante el almacenamiento la firmeza de los frutos disminuyera gradualmente. La variedad 'Osteen' presentó una firmeza comercial hasta el día 3, sin tener diferencia significativa con 'Fabián'; ambos materiales se consideraron como los de menor vida de anaquel en función de su firmeza. Para una calidad aceptable de consumo de los mangos en relación con su firmeza, éstos deberán de presentar valores mínimos de 1.75 a $2.0 \mathrm{~kg}$ $\mathrm{cm}^{-2}$ según Mitcham y McDonald (1992). En general, la mayoría de los frutos lograron mantener la firmeza por un tiempo de $9 \mathrm{~d}$, hasta $12 \mathrm{~d}$ en los mangos 'Ah-Ping', 'Manila Rosa' y 'Kent'.

Es importante hacer notar que los frutos de 'Fabián' presentaron en esta temporada de evaluación y en la reportada por Siller-Cepeda et al. (1994) valores de firmeza por debajo del mínimo aceptable, lo que podría indicar que este cultivar debió clasificarse y cosecharse dentro de la categoría de madurez intermedia, aunque sus características de corte no estaban bien definidas para esa etapa de desarrollo. Esta discusión es acorde con lo reportado por Mosqueda et al. (1996), quienes lo agruparon en madurez intermedia junto con 'Tommy 'Atkins'.

\section{Color de pulpa y cáscara}

En todos los mangos el color de pulpa al momento de corte fue amarillo claro, con valores promedios de luminosidad y ángulo de matiz de $76 \pm 8$ y $93 \pm 7$, respectivamente (Cuadro 3). Los mangos 'Ah-Ping', 'Kent' y 'Fabián' mostraron la tonalidad de pulpa más anaranjada al momento de corte con ${ }^{\circ} \mathrm{Hue} \leq 86$. Durante el almacenamiento estas variedades también desarrollaron el color más atractivo, pero sin diferencia significativa con 'Van Dyke'. Los mangos 'Edward' no presentaron el cambio de color anaranjado característico en la pulpa, sino únicamente ligeros cambios en el ángulo de matiz de 98 a 90, con tonalidades amarillentas, al igual que 'Osteen'.

Las variedades de mango, con excepción de 'Keitt' y 'Diplomático', desarrollaron tonalidades de color anaranjadas, amarillas y rojas en la epidermis, que fueron más intensas y atractivas en las variedades 'Ah-Ping', 'Van Dyke', 'Manila Rosa' y 'Tommy Atkins', con valores de alrededor de $70{ }^{\circ}$ Hue (Cuadro 4). Los mangos 'Keitt' únicamente mostraron un cambio de color de piel de verde oscuro a verde-amarillo $\left({ }^{\circ} \mathrm{Hue}=95\right)$, similar al observado en los frutos 'Diplomático' $\left({ }^{\circ} \mathrm{Hue}=89\right)$ aunque con un amarillo más intenso. Según Campbell (1992), el color externo de los frutos de mango no debería considerarse como factor de calidad ya que se pueden presentar variedades de mango que no desarrollan un color atractivo en la cáscara, pero sí un excelente sabor, como 'Keitt' .

Cuadro 2. Firmeza (kgf) en frutos de mango de tres grupos de maduración expuestos bajo simulación de mercadeo por $12 \mathrm{~d}$ a $20{ }^{\circ} \mathrm{C} .{ }^{\dagger}$

\begin{tabular}{|c|c|c|c|c|c|}
\hline \multirow{2}{*}{ Cultivar } & \multicolumn{5}{|c|}{ Días de almacenamiento a $20^{\circ} \mathrm{C}$} \\
\hline & 0 & 3 & 6 & 9 & 12 \\
\hline \multicolumn{6}{|c|}{ Maduración temprana } \\
\hline 'Edward' & $13.0 \pm 1.3 \mathrm{~b}^{\dagger \dagger}$ & $12.2 \pm 1.2 \mathrm{a}$ & $5.6 \pm 1.0 \mathrm{~b}$ & $3.0 \pm 0.6 \mathrm{a}$ & $0.7 \pm 0.2 \mathrm{c}$ \\
\hline ‘Diplomático' & $8.2 \pm 1.1 \mathrm{c}$ & $8.0 \pm 1.0 \mathrm{~b}$ & $3.5 \pm 0.8 \mathrm{c}$ & $1.3 \pm 0.3 \mathrm{~b}$ & $0.6 \pm 0.1 \mathrm{c}$ \\
\hline 'Ah-Ping' & $10.8 \pm 1.6 \mathrm{bc}$ & $6.6 \pm 0.9 \mathrm{~b}$ & $4.4 \pm 0.8 \mathrm{bc}$ & $3.6 \pm 0.7 \mathrm{a}$ & $2.3 \pm 0.3 \mathrm{a}$ \\
\hline 'Van Dyke' & $17.0 \pm 1.7 \mathrm{a}$ & $12.5 \pm 1.2 \mathrm{a}$ & $7.4 \pm 0.9 \mathrm{ab}$ & $3.3 \pm 0.6 \mathrm{a}$ & $1.7 \pm 0.2 \mathrm{~b}$ \\
\hline 'Haden’' & $13.9 \pm 1.5 \mathrm{~b}$ & $13.6 \pm 1.3 \mathrm{a}$ & $8.2 \pm 1.0 \mathrm{a}$ & $1.8 \pm 0.3 \mathrm{~b}$ & $1.6 \pm 0.3 \mathrm{~b}$ \\
\hline \multicolumn{6}{|c|}{ Maduración intermedia } \\
\hline ‘Manila Rosa’ & $14.0 \pm 2.2 \mathrm{ab}$ & $11.0 \pm 1.6 \mathrm{~b}$ & $6.5 \pm 1.2 \mathrm{ab}$ & $2.0 \pm 0.5 \mathrm{~b}$ & $1.8 \pm 0.4 \mathrm{a}$ \\
\hline 'Tommy Atkins' & $12.8 \pm 1.2 \mathrm{a}$ & $10.1 \pm 1.5 \mathrm{~b}$ & $6.2 \pm 1.2 \mathrm{~b}$ & $3.2 \pm 0.9 \mathrm{ab}$ & $0.6 \pm 0.2 \mathrm{~b}$ \\
\hline 'Kent' & $18.5 \pm 2.4 \mathrm{a}$ & $16.2 \pm 1.7 \mathrm{a}$ & $9.1 \pm 1.5 \mathrm{a}$ & $5.9 \pm 1.8 \mathrm{a}$ & $2.6 \pm 0.5 \mathrm{a}$ \\
\hline \multicolumn{6}{|c|}{ Maduración tardía } \\
\hline ‘Osteen’ & $10.1 \pm 1.2 \mathrm{a}$ & $5.3 \pm 1.6 \mathrm{~b}$ & $1.3 \pm 0.5 \mathrm{c}$ & $1.0 \pm 0.2 \mathrm{~b}$ & $0.7 \pm 0.1 \mathrm{~b}$ \\
\hline 'Palmer' & $12.5 \pm 2.2 \mathrm{a}$ & $11.9 \pm 1.3 \mathrm{a}$ & $4.6 \pm 1.0 \mathrm{~b}$ & $1.1 \pm 0.3 b$ & $1.0 \pm 0.2 \mathrm{ab}$ \\
\hline ‘Fabián’' & $6.7 \pm 1.8 \mathrm{~b}$ & $4.0 \pm 1.4 \mathrm{~b}$ & $1.7 \pm 0.5 \mathrm{c}$ & $1.1 \pm 0.4 \mathrm{~b}$ & $0.7 \pm 0.1 b$ \\
\hline 'Keitt' & $14.0 \pm 2.0 \mathrm{a}$ & $9.7 \pm 1.0 \mathrm{a}$ & $7.0 \pm 1.2 \mathrm{a}$ & $2.2 \pm 0.5 \mathrm{a}$ & $1.2 \pm 0.3 \mathrm{a}$ \\
\hline
\end{tabular}

${ }^{\dagger}$ Media y desviación estándar de 10 frutos. ${ }^{\dagger \dagger}$ Medias con letras iguales por columna y grupo de maduración no son estadísticamente diferentes (Tukey, 0.05). 
Cuadro 3. Componentes del color (luminosidad, ángulo de matiz y cromaticidad) en la pulpa de frutos de mango de tres grupos de maduración, expuestos a simulación de mercadeo por $12 \mathrm{~d}$ a $20{ }^{\circ} \mathrm{C}$.

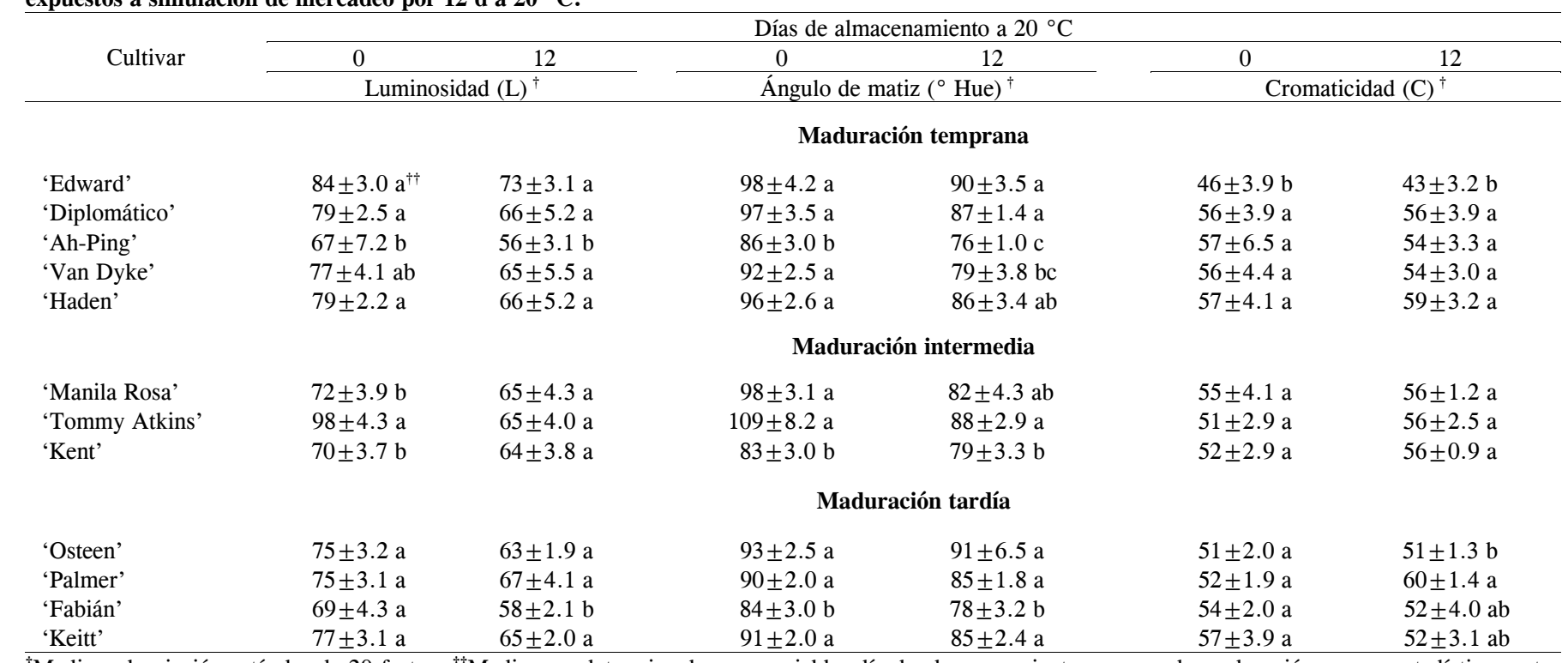

${ }^{\dagger}$ Media y desviación estándar de 20 frutos. ${ }^{\dagger}$ Medias con letras iguales por variable, día de almacenamiento y grupo de maduración no son estadísticamente diferentes (Tukey, 0.05).

Cuadro 4. Componentes del color (luminosidad, ángulo de matiz y cromaticidad) en la cáscara de frutos de mango de tres grupos de maduración, expuestos bajo simulación de mercadeo por $12 \mathrm{~d}$ a $20{ }^{\circ} \mathrm{C}$.

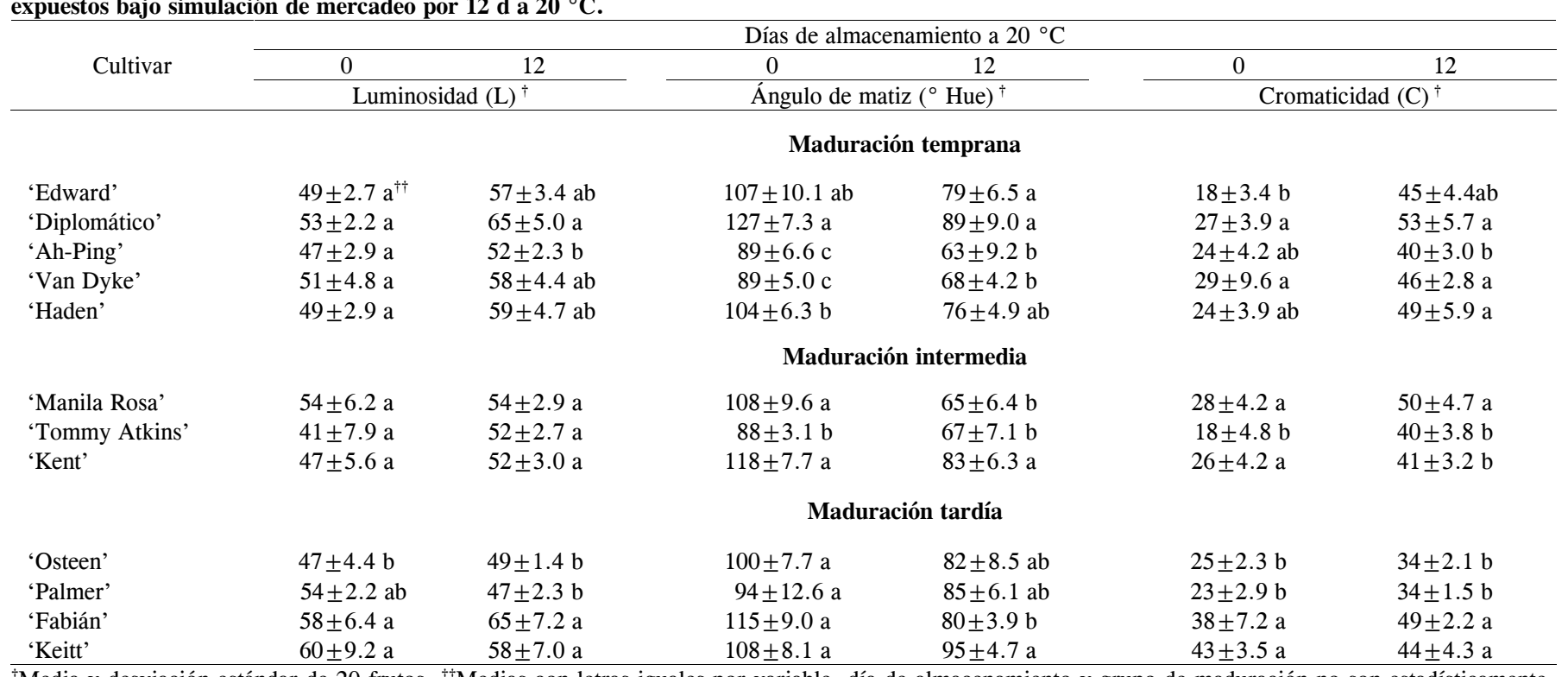

${ }^{\top}$ Media y desviación estándar de 20 frutos. ${ }^{\dagger}$ Medias con letras iguales por variable, día de almacenamiento y grupo de maduración no son estadísticamente diferentes (Tukey, 0.05).

\section{pH y acidez titulable}

Los mangos de maduración temprana mostraron valores de $\mathrm{pH}$ bajos y acidez titulable alta, con 'Edward' como el más ácido con $2.2 \%$ y pH 2.8 al día de corte (Cuadro 5). Estos valores no corresponden a frutos inmaduros, ya que la firmeza y el color fueron aceptables (Cuadro 2 y 3). Al final del almacenamiento la acidez disminuyó y el pH aumentó en este cultivar, al igual que en los demás. Los frutos de 'Diplomático', 'Manila Rosa' y 'Fabián' mostraron valores altos de acidez (> $1.0 \%)$, que disminuyeron entre 60 y $90 \%$ durante el almacenamiento. Los demás materiales exhibieron menores porcentajes de acidez a la cosecha, con valores similares entre ellos al final del estudio.

Gutiérrez et al. (1996) reportaron un descenso drástico en la acidez de mango 'Manila' de 2.7 a $0.2 \%$, similar al comportamiento de 'Manila Rosa'. Lakshminarayana (1980) indica que los mangos 'Keitt' e 'Irwin' en madurez 
de consumo alcanzaban valores de $\mathrm{pH}$ de $4.5 \mathrm{y}$ acidez de $0.12 \%$. Este mismo autor menciona que la acidez de los frutos se reduce durante la maduración, ya que en mangos 'Haden' y 'Kent' en madurez fisiológica los valores fueron de 0.97 y $0.51 \%$, respectivamente, y de 0.31 y 0.49 $\%$ en madurez de consumo. Lebrun et al. (2008) también estudiaron la maduración de mangos 'Keitt', 'Kent' y 'Cogshall' cosechados en diferentes estados de desarrollo, y en todos los materiales observaron pérdida de la acidez e incremento de los sólidos solubles totales. Similares resultados se encontraron en este estudio, aunque los frutos 'Haden', 'Kent' y 'Osteen' permanecieron con características ácidas al final del almacenamiento, lo que podría favorecer un mayor periodo de comercialización.

\section{Sólidos solubles totales (SST)}

Los mangos de madurez temprana presentaron los valores más altos de SST al momento de corte con $7.7^{\circ}$ Brix en promedio; destaca 'Ah-Ping' con $10.4{ }^{\circ} \mathrm{Brix}$, sin estar asociado con madurez adelantada, por sus valores de firmeza (Cuadro 2) y por desarrollar $13.2{ }^{\circ}$ Brix durante el almacenamiento (Cuadro 5).

Según Báez (1998), los mangos 'Tommy Atkins', 'Haden' y 'Kent' con características de exportación deben presentar al momento de corte valores mínimos de 7.3 ${ }^{\circ}$ Brix, y en el presente trabajo algunos valores fueron ligeramente superiores. Todas las variedades presentaron un incremento en SST satisfactorio durante el almacenamiento. Lakshminarayana (1980) reportó un incremento de 9.8 a $17{ }^{\circ}$ Brix en frutos 'Haden' y de 8.7 a $19.4{ }^{\circ}$ Brix en 'Kent', ambos con características muy dulces. Gutiérrez et al. (1996) en frutos 'Manila' observaron valores de 8.5 a $14.6{ }^{\circ}$ Brix durante simulación de mercadeo a 23 ${ }^{\circ} \mathrm{C}$.

\section{Relación azúcar/ácido (RAA)}

Al día de corte todos los materiales presentaron una baja RAA (datos no mostrados), característica de alta acidez y bajo SST. Sin embargo, conforme los frutos maduraron tuvieron un aumento en RAA debido a la hidrólisis de carbohidratos y a la disminución de los ácidos orgánicos requeridos en el proceso de maduración del fruto (Mitra y Baldwin, 1997).

En general, las variedades de mango producidas en Sinaloa no presentaron relaciones elevadas de RAA (Cuadro 5), pues únicamente 'Diplomático' y 'Keitt' desarrollaron valores superiores a 100 (frutos con menor acidez). Los frutos de 'Ah-Ping', 'Van Dyke' y 'Tommy Atkins' presentaron valores promedio menores de RAA (69), que se asocian con frutos menos dulces. La baja RAA para mangos 'Kent' (23.6), 'Haden' (32.3) y 'Osteen' (23.3) se puede deber a que el tiempo de estudio no fue suficiente para que los frutos alcanzaran su madurez comercial completa, por lo que su almacenamiento y vida de anaquel podrían prolongarse e incrementar la RAA. En mango el incremento de RAA se asocia con la degradación de carbohidratos complejos como celulosa, pectinas y hemicelulosa, a monosacáridos como glucosa y fructosa (Tharanathan et al., 2006), lo que ayuda a acentuar el dulzor de los frutos.

Cuadro 5. Características químicas de calidad en la pulpa de frutos de mango de tres grupos de maduración, expuestos por $12 \mathrm{~d}$ a $20{ }^{\circ} \mathrm{C}$.

\begin{tabular}{|c|c|c|c|c|c|c|c|}
\hline \multirow{3}{*}{ Cultivar } & \multicolumn{7}{|c|}{ Días de almacenamiento a $20^{\circ} \mathrm{C}$} \\
\hline & 0 & 12 & 0 & 12 & 0 & 12 & 12 \\
\hline & \multicolumn{2}{|c|}{$\mathrm{pH}^{\dagger}$} & \multicolumn{2}{|c|}{$\begin{array}{c}\text { Acidez titulable } \\
(\% \text { ácido cítrico })^{\dagger}\end{array}$} & \multicolumn{2}{|c|}{$\operatorname{SST}\left({ }^{\circ} \text { Brix }\right)^{\dagger}$} & $\begin{array}{c}\text { Relación } \\
\text { Azúcar/ácido }\end{array}$ \\
\hline & \multicolumn{7}{|c|}{ Maduración temprana } \\
\hline 'Edward' & $2.8 \pm 0.2 \mathrm{a}^{\dagger \dagger}$ & $3.6 \pm 0.1 \mathrm{~b}$ & $2.2 \pm 0.3 \mathrm{a}$ & $0.3 \pm 0.5 \mathrm{a}$ & $5.3 \pm 1.6 \mathrm{~b}$ & $16.8 \pm 1.0 \mathrm{a}$ & $56.0 \pm 3.2 \mathrm{c}$ \\
\hline ‘Diplomático’ & $3.2 \pm 0.1 \mathrm{a}$ & $4.1 \pm 0.3 \mathrm{a}$ & $1.1 \pm 0.2 \mathrm{~b}$ & $0.1 \pm 0.1 \mathrm{c}$ & $6.8 \pm 1.0 \mathrm{~b}$ & $14.8 \pm 1.2 \mathrm{~b}$ & $134.5 \pm 9.2 \mathrm{a}$ \\
\hline 'Ah-Ping' & $3.6 \pm 0.1 \mathrm{a}$ & $4.8 \pm 0.2 \mathrm{a}$ & $0.9 \pm 0.2 \mathrm{a}$ & $0.2 \pm 0.1 \mathrm{~b}$ & $10.4 \pm 2.0 \mathrm{a}$ & $13.2 \pm 1.0 \mathrm{~b}$ & $66.0 \pm 2.4 \mathrm{~b}$ \\
\hline 'Van Dyke' & $3.0 \pm 0.1 \mathrm{a}$ & $4.1 \pm 0.1 \mathrm{ab}$ & $0.9 \pm 0.1 \mathrm{~b}$ & $0.2 \pm 0.1 \mathrm{~b}$ & $9.9 \pm 1.8 \mathrm{a}$ & $16.3 \pm 1.3 \mathrm{a}$ & $70.4 \pm 1.4 \mathrm{~b}$ \\
\hline \multirow[t]{2}{*}{ ‘Haden’ } & $2.9 \pm 0.2 \mathrm{a}$ & $3.9 \pm 0.1 \mathrm{a}$ & $0.8 \pm 0.1 \mathrm{c}$ & $0.5 \pm 0.2 \mathrm{a}$ & $6.4 \pm 1.0 \mathrm{~b}$ & $15.2 \pm 1.5 \mathrm{ab}$ & $32.3 \pm 2.1 \mathrm{~d}$ \\
\hline & \multicolumn{7}{|c|}{ Maduración intermedia } \\
\hline ‘Manila Rosa' & $3.4 \pm 0.1 \mathrm{a}$ & $4.7 \pm 0.3 \mathrm{a}$ & $1.1 \pm 0.2 \mathrm{a}$ & $0.2 \pm 0.1 \mathrm{~b}$ & $5.8 \pm 2.0 \mathrm{a}$ & $11.7 \pm 1.0 \mathrm{~b}$ & $46.8 \pm 8.2 \mathrm{~b}$ \\
\hline 'Tommy Atkins' & $3.2 \pm 0.2 \mathrm{a}$ & $4.3 \pm 0.3 \mathrm{a}$ & $0.3 \pm 0.1 \mathrm{~b}$ & $0.2 \pm 0.1 \mathrm{~b}$ & $6.0 \pm 1.6 \mathrm{a}$ & $13.9 \pm 1.7 \mathrm{a}$ & $69.5 \pm 5.1 \mathrm{a}$ \\
\hline \multirow[t]{2}{*}{ 'Kent' } & $4.7 \pm 0.2 \mathrm{a}$ & $4.9 \pm 0.3 \mathrm{a}$ & $0.5 \pm 0.2 b$ & $0.6 \pm 0.1 \mathrm{a}$ & $4.1 \pm 0.2 \mathrm{~b}$ & $13.7 \pm 1.8 \mathrm{a}$ & $23.6 \pm 6.7 \mathrm{c}$ \\
\hline & \multicolumn{7}{|c|}{ Maduración tardía } \\
\hline 'Osteen' & $3.4 \pm 0.2 \mathrm{ab}$ & $4.6 \pm 0.2 \mathrm{a}$ & $0.6 \pm 0.1 \mathrm{~b}$ & $0.5 \pm 0.2 \mathrm{a}$ & $6.1 \pm 4.4 \mathrm{ab}$ & $12.6 \pm 0.8 \mathrm{~b}$ & $23.3 \pm 2.2 \mathrm{c}$ \\
\hline 'Palmer' & $3.2 \pm 0.1 \mathrm{a}$ & $4.9 \pm 0.3 \mathrm{a}$ & $0.8 \pm 0.1 \mathrm{~b}$ & $0.3 \pm 0.1 \mathrm{~b}$ & $7.2 \pm 4.4 \mathrm{a}$ & $14.7 \pm 1.4 \mathrm{a}$ & $54.4 \pm 3.1 \mathrm{~b}$ \\
\hline ‘Fabián’ & $3.2 \pm 0.1 \mathrm{~b}$ & $4.7 \pm 0.2 \mathrm{a}$ & $1.0 \pm 0.2 \mathrm{a}$ & $0.4 \pm 0.1 \mathrm{a}$ & $6.4 \pm 4.4 \mathrm{a}$ & $12.6 \pm 1.3 \mathrm{~b}$ & $28.6 \pm 4.9 \mathrm{c}$ \\
\hline ‘Keitt’' & $3.8 \pm 0.1 \mathrm{a}$ & $4.7 \pm 0.3 \mathrm{a}$ & $0.5 \pm 0.1 \mathrm{~b}$ & $0.1 \pm 0.1 \mathrm{c}$ & $5.8 \pm 4.4 \mathrm{~b}$ & $14.0 \pm 1.4 \mathrm{a}$ & $100.0 \pm 8.8 \mathrm{a}$ \\
\hline
\end{tabular}

${ }^{\dagger}$ Media y desviación estándar de 10 repeticiones. ${ }^{\dagger}$ Medias con letras iguales por columna, día de evaluación y época de maduración no son estadísticamente diferentes (Tukey, 0.05). SST = sólidos solubles totales. 
Lakshminarayana (1980) reportó que los mangos 'Keitt' y 'Haden' presentan una RAA de 167 y 85 respectivamente, superiores a los obtenidos en este trabajo. Manzano et al. (1993) señalaron resultados similares a este estudio para frutos de mango 'Haden' con una RAA de 37.5 cuando fueron almacenados por $20 \mathrm{~d}$ a $20^{\circ} \mathrm{C}$.

\section{Respiración}

Todos los frutos mostraron una producción de $\mathrm{CO}_{2}$ característica de frutos climatéricos, con la máxima tasa respiratoria entre los 8 y $10 \mathrm{~d}$ de almacenamiento (datos no mostrados). 'Edward' y 'Kent' alcanzaron $71 \mathrm{~mL} \mathrm{CO}$ $\mathrm{kg}^{-1} \mathrm{~h}^{-1}$, mientras que 'Tommy Atkins', 'Palmer' y 'Osteen' registraron un promedio de $40 \mathrm{~mL} \mathrm{CO} \mathrm{kg}^{-1} \mathrm{~h}^{-1}$ (Cuadro 6). Era de esperarse que los frutos de menor actividad respiratoria mostraran una mayor vida de anaquel; sin embargo, esto no se observó ya que los valores de firmeza fueron bajos al final del estudio en los frutos de menor actividad respiratoria. Esto posiblemente se deba a diferencias fisiológicas y en composición de los materiales estudiados.

Cuadro 6. Valores mínimos y máximos de respiración en frutos de mango de tres grupos de maduración, durante $12 \mathrm{~d} \mathrm{a} 20{ }^{\circ} \mathrm{C} .{ }^{\dagger}$

\begin{tabular}{|c|c|c|}
\hline \multirow[t]{2}{*}{ Cultivar } & \multicolumn{2}{|c|}{$\begin{array}{l}\text { Rango de respiración } \\
\left(\mathrm{mL} \mathrm{CO}_{2} \mathrm{~kg}^{-1} \mathrm{~h}^{-1}\right)\end{array}$} \\
\hline & \multicolumn{2}{|c|}{ Maduración temprana } \\
\hline 'Edward’ & $23 \pm 2.1 \mathrm{~b}^{\dagger \dagger}$ & $71 \pm 5.0 \mathrm{a}$ \\
\hline 'Diplomático' & $22 \pm 3.3 \mathrm{~b}$ & $54 \pm 8.7 \mathrm{~b}$ \\
\hline 'Ah-Ping' & $39 \pm 5.0 \mathrm{a}$ & $58 \pm 4.1 \mathrm{~b}$ \\
\hline 'Van Dyke' & $32 \pm 4.6 \mathrm{a}$ & $65 \pm 3.6 \mathrm{ab}$ \\
\hline \multirow[t]{2}{*}{ 'Haden' } & $20 \pm 1.8 \mathrm{~b}$ & $58 \pm 3.5 \mathrm{~b}$ \\
\hline & \multicolumn{2}{|c|}{ Maduración intermedia } \\
\hline 'Manila Rosa' & $21 \pm 1.2 \mathrm{~b}$ & $50 \pm 3.1 \mathrm{~b}$ \\
\hline 'Tommy Atkins' & $18 \pm 2.2 \mathrm{~b}$ & $42 \pm 5.0 \mathrm{~b}$ \\
\hline 'Kent' & $35 \pm 3.9 \mathrm{a}$ & $71 \pm 4.5 \mathrm{a}$ \\
\hline
\end{tabular}

Maduración tardía

\begin{tabular}{lll} 
'Osteen' & $14 \pm 0.8 \mathrm{~b}$ & $39 \pm 2.1 \mathrm{~b}$ \\
'Palmer' & $15 \pm 1.0 \mathrm{~b}$ & $40 \pm 2.5 \mathrm{~b}$ \\
'Fabián' & $29 \pm 2.3 \mathrm{a}$ & $53 \pm 4.1 \mathrm{a}$ \\
'Keitt' & $15 \pm 1.1 \mathrm{~b}$ & $54 \pm 3.9 \mathrm{a}$ \\
\hline
\end{tabular}

${ }^{\dagger}$ Media y desviación estándar de tres repeticiones. ${ }^{\dagger}$ Medias con letras iguales por rango de valuación y época de maduración no son estadísticamente diferentes (Tukey, 0.05).

La máxima producción de $\mathrm{CO}_{2}$ aquí encontrada coincide con algunos artículos en cuanto a que máximas concentraciones ocurren entre 7 y $11 \mathrm{~d}$ de almacenamiento en condiciones de simulación de mercadeo (Mitra y Balwin, 1997). Para mangos a $20{ }^{\circ} \mathrm{C}$, Kader (1997) reportó un rango de 35 a $85 \mathrm{~mL} \mathrm{CO}_{2} \mathrm{~kg}^{-1} \mathrm{~h}^{-1}$, ligeramente superior al encontrado en este estudio.

\section{CONCLUSIONES}

Los frutos de mango de variedades con diferentes etapas de maduración desarrollaron características de calidad excelentes para el consumidor, reflejados en buena firmeza, color, acidez y sólidos solubles totales, con excepción de 'Diplomático' y 'Fabián'. La velocidad de respiración fue moderada, lo que permite que los frutos tengan una vida de anaquel entre 8 y 12 d. En general, 'Palmer', 'Edward' y 'Ah-Ping' son variedades con características de calidad competitiva, si se comparan con los mangos 'Kent', 'Keitt', 'Tommy Atkins' y 'Haden' que se destinan al mercado de exportación, mientras que 'Diplomático' podría competir con frutos pequeños como 'Manila'.

\section{BIBLIOGRAFÍA}

AOAC, Association of Official Analytical Chemists (1998) Official Methods of Analysis. 16th ed. S William (ed). Published by Association of Official Analytical Chemists. Washington, D.C. USA. CD-ROM.

Báez M A, J Siller, B Heredia, E Araiza, R S García, M D Muy (1997) Fisiología poscosecha de frutos de chicozapote (Achras sapota L.) durante condiciones de mercadeo. Proc. Interam. Soc. Trop. Hort. 41:209-214.

Báez S R (1998) Norma Mexicana de Calidad para Mango Fresco de Exportación. Comité Técnico Científico de Empacadores de Mango de Exportación, A. C. (EMEX, A. C.). Guadalajara, Jalisco. México. $4 \mathrm{p}$.

Bourne M C (1980) Texture evaluation of horticultural crops. HortScience 15:51-57.

Campbell R (1992) Mangos, a Guide to Mangos in Florida. Fairchild Tropical Garden. Miami, Florida. USA. 90 p.

FAO, Food and Agriculture Organization (2004) Agricultural data. FAOSTAT. http://www.faostat.org. (26/11/2004).

Gutiérrez B, J Cruz, K Parkin, H Galindo (1996) Effect of refrigerated storage on manila mangoes (Mangifera indica L.) after hydrothermal treatment. Acta Hort. 455:679-686.

Hidalgo M, J Cruz, K Parkin, H Galindo (1996) Refrigerated storage and chilling injury development of manila mangoes (Mangifera indica L.). Acta Hort. 455:718-725.

Hofman P J, L Smith, D Joyce, G Johnson, G Meiburg (1997) Bagging of mango (Mangifera indica $\mathrm{cv}$. 'Keitt') influences fruit quality and mineral composition. Postharv. Biol. Technol. 12:83-91.

Ireta O A, E C Guzmán (2002) Guía Técnica para la Producción de Mango en Sinaloa. Instituto Nacional de Investigaciones Forestales, Agrícolas y Pecuarias. Folleto Técnico 22. Culiacán, Sinaloa. $152 \mathrm{p}$.

Kader A A (1997) Perishables Handling Produce Facts Mango. In: Recommendations for Maintaining Postharvest Quality. Newsletter \# 89. University of California, USA. 4 p.

Lakshminarayana S (1980) Mango. In: Tropical and Subtropical Fruits. S Nagy, P E Shaw (eds). AVI Publishing CT. USA. pp:184-257.

Lebrun M, A Plotto, K Goodner, M Ducampa, E Baldwin (2008) Discrimination of mango fruit maturity by volatiles using the electronic nose and gas chromatography. Postharv. Biol. Technol. 48:122-131.

Manzano J, E Pérez, E Rojas (1993) Coating waxes on Haden mango fruits (Mangifera indica L.) cultivars for export. Acta Hort. 455:738-746. 
MINITAB (2004) Statistical software. Release number 14.0. www.minitab.com.

Mitcham E J, R E McDonald (1992) Cell wall modification during ripening of 'Keitt' and 'Tommy Atkins' mango fruit. J. Amer. Soc. Hort. Sci. 117:912-924.

Mitra S K, E A Baldwin (1997) Mango. In: Postharvest Physiology and Storage of Tropical and Subtropical Fruits. S K Mitra (ed). CAB International. New York, USA. pp:85-122.

Mosqueda R V, F Santos, L E Becerra, Z D Ortega, A Ángel (1996) Manual para Cultivar Mango en la Planicie Costera del Golfo de México. Instituto Nacional de Investigaciones Forestales, Agrícolas y Pecuarias. Folleto Técnico 15. pp:39-40.

Muy R D, J Siller, J Díaz, B Valdez (2004) Las condiciones de almacenamiento y el encerado afectan el estado hídrico y la calidad de mango. Rev. Fitotec. Mex. 27:201-209.

Osuna-García J A, M L Guzmán-Robles, B Tovar-Gómez, M MataMontes de Oca, V A Vidal-Martínez (2002) Calidad del mango 'Ataulfo' producido en Nayarit, México. Rev. Fitotec. Mex. 25:367-374.

SIAP, Servicio de Información Agroalimentaria y Pesquera (2008) Secretaría de Agricultura, Ganadería, Desarrollo Rural, Pesca y Alimentación (SAGARPA). Producción Agrícola 2006. http://www.siap.gob.mx/aagricola_siap/ientidad/index.jsp $(23 / 04 / 2008)$.

SICM, Sistema de Información Comercial Mexicano (2008) Consumo nacional aparente y consumo per capita de mango. http://w4.siap.sagarpa.gob.mx (9/06/2008).

Siller-Cepeda J, D Muy, E Araiza, M Báez, J Rodríguez, R Báez, A Ireta (1994) Evaluación de la calidad de clones de mango introducidos a Sinaloa. Proc. Interam. Soc. Trop. Hort. 38:3742.

Tharanathan R N, H M Yashoda, T N Prabha (2006) Mango (Mangifera indica L.), "the king of fruits"-an overview. Food Rev. Int. 22:95-123. 